\title{
Transversalidad educativa: casos clínicos para el estudio de Geriatría y Patologías Osteoarticulares y su intervención en Autonomía e Independencia Funcional en el Adulto
}

\author{
Jimenez Arberas, Estíbaliza , Sánchez Menéndez, Marta María b y Ruiz Fernández, \\ María Luisa ${ }^{\mathrm{c}}$

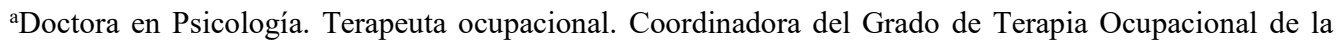 \\ Facultad Padre Ossó, Universidad de Oviedo; estibaliz@facultadpadreosso.es boctora por la \\ Universidad de Oviedo. Médico Especialista en Reumatología. Servicio de Reumatología Centro Médico \\ de Asturias; msanchezmene@gmail.com. ${ }^{c}$ Doctora por la Universidad de Oviedo. Médico Especialista \\ en Medicina de la Educación Física y el Deporte. Profesora- coordinadora de Anatomía Humana y \\ Geriatría y Patologías Osteoarticulares. Grado Terapia Ocupacional. Facultad Padre Ossó. Universidad \\ de Oviedo; luisa@facultadpadreosso.es
}

\section{Abstract}

The aim of this project is to introduce the students into the knowledge of the discapabilities trough the cases studies related with two different subjects, Geriatrics and Osteoarticular Pathologies and Autonomy and Functional Indepence in the Adult, for this purpose, students were provided with enough material for the resolution of any case studies simulated situation in the GOP lessons and in the AFI lessons for developping intervention strategies based on the fundamentals of Occupational Therapy.

Five different clinical cases were proposed in reumatology and other four in traumatology. In AFI one of each discipline case was chosen, on the specific they were, rheumatoid hand and amputation. The methodological innovation of this project is the educational transversality. To evaluate this skills a gammification was created.

Keywords: Occupational Therapy, study cases, innovation, gammification, cross discipline

\begin{abstract}
Resumen
El objetivo de este proyecto es introducir al alumnado en el conocimiento de la discapacidad a través de casos clínicos en dos asignaturas, Geriatría y Patologías Osteoarticulares (POA) y Autonomía e Independencia Funcional en el Adulto (AIF), para ello dispusieron de material y tutorización para la resolución de los casos durante las clases de POA, por otro lado en AIF desarrollan estrategias de intervención desde la Terapia Ocupacional. Se prepararon cinco casos clínicos en reumatología (artritis reumatoide, colagenosis, espondiloartropatías, artritis cristalinas e infecciosas) y cuatro en traumatología (tendinopatías, artrosis, fracturas y amputaciones). En AIF se eligieron dos casos, mano reumatoide más específicamente un caso clínico de artritis reumatoide y otro caso clínico, una amputación de mano. La innovación metodológica de este poyecto es la transversalidad educativa. Se diseño finalmente una gamificación para la evaluación de conocimientos realizando un scape room.
\end{abstract}

Palabras claves: Casos clínicos, transversalidad, Terapia Ocupacional, innovación, gamificación

\section{Introducción}


Este proyecto toma como referencia la Clasificación Internacional del Funcionamiento de la Discapacidad y la Salud (CIF) (WHO, 2001), la cual clasifica en cinco dimensiones el funcionamiento del ser humano: 1) funciones corporales-estructuras corporales, 2) actividades, 3) participación, 4) factores contextuales y 5) factores personales. Nuestro proyecto engloba todas las dimensiones ya que precisa del conocimiento de las funciones y estructuras corporales (vendaje neuromuscular, vendajes, tabla canadiense, etc.), las actividades de la vida diaria, la participación social, factores ambientales donde incluiríamos los productos de apoyo, etc. Para los terapeutas ocupacionales la CIF incluye todas aquellas actividades, tareas y roles que están dentro de las competencias profesionales. Son muchos los autores que han discutido el uso potencial de la CIF en medidas de resultados (Arthanat \& Lenker, 2004; Lenker \& Jutai, 2002) y son además muchos los instrumentos para la selección de PA que incluyen los componentes de la CIF (Scherer \& Sax, 2005) porque proporciona un esquema sistemático para la organización de necesidades y capacidades de los clientes tales como el de priorizar objetivos porque la CIF presta por un lado atención al funcionamiento y la discapacidad y por otro a los factores contextuales (Cushman \& Scherer, 1996; Jutai, Fuhrer, Demers, Scherer, \& DeRuyter, 2005; Hubert, 2003; Scherer, Jutai, Fuhrer, Scherer \& Glueckauf, 2005), factores no incluidos en otros modelos, incluso describe la relación entre las condiciones de salud, actividades habituales y la participación en la sociedad teniendo en cuenta estos factores (además de los personales) (WHO, 2001). Desde la Sociedad Española de Reumatología (SER) se da gran relevancia al estudio y discusión de casos clínicos, y prueba de ello son las secciones de casos clínicos publicados en su revista Reumatología Clínica, año 2018. Creemos que esto avala y refuerza la necesidad de adecuar nuestros métodos didácticos y trabajar desde la Facultad la parte práctica de la Terapia Ocupacional. Además, el College of Occupational Therapists considera a la CIF un cambio de perspectiva de solo un diagnóstico médico a tener en cuenta los problemas del individuo a partir de su contexto (COT, 2004), de lo que se deriva la importancia de aunar diferentes asignaturas para conseguir una perspectiva holística en el tratamiento y centrarnos en un modelo bio-psico-social. Paralelamente, en el programa de atención tutorial y en la Encuesta General de Enseñanza (PATTO curso 2016-2017), se incluye por parte del alumnado en los resultados recogidos tanto la necesidad de conocimientos y habilidades practicas a la hora de desarrollar sus competencias profesionales como la implementación de las mismas en todo el trayecto curricular.

\section{Objetivos}

2.1. Implementar una metodología que permita adquirir habilidades y competencias profesionales, basada en casos clínicos reales para la adquisición de competencias específicas.

2.2. Innovar en la coordinación docente transversal entre asignaturas complementarias.

\section{Desarrollo de la innovación.}

\subsection{Plan de Trabajo desarrollado}

$\mathrm{Al}$ inicio del curso se explicó al alumnado el modelo didáctico de la asignatura consistente en el desarrollo de casos clínicos. En la plataforma virtual (moodle) se alojó el material necesario para la resolución de los casos clínicos, poniendo a su disposición material bibliográfico y presentaciones en PowerPoint acorde al temario establecido en la guía docente. Se entregaron, siempre en la plataforma virtual, dos grupos de casos clínicos: a) casos reumatológicos (artritis reumatoide, colagenosis, espondiloartropatías, artritis cristalinas e infecciosas), b) casos traumatológicos (tendinopatías, artrosis, fracturas y amputaciones). 
En AIF en el Adulto se entregaron, siempre en la plataforma virtual, protocolos de actuación e intervención terapéutica, técnicas de exploración en Terapia Ocupacional y materiales de apoyo.

Se tomaron como indicadores las calificaciones obtenidas en las convocatorias ordinarias y el grado de satisfacción del alumnado, para lo cual se diseñó un cuestionario. Se adjuntan los indicadores y el cuestionario en el epígrafe de resultados

\subsection{Descripción de la Metodología}

\subsubsection{Descripción de la Metodología en Geriatría y Patologías Osteroarticulares}

Cada caso clínico incluía imágenes de una región anatómica con patología identificable (derrame articular, signos flogóticos, equímosis, hematomas, etc.), historia clínica del paciente incluyendo datos personales (sexo, edad, profesión, grado de ocupación, etc.), motivo de consulta y síntomas descritos por el paciente, incluido el dolor y tipo del mismo. Así mismo se exponían los signos clínicos de la exploración física y las pruebas complementarias, tanto de imágenes como de laboratorio (Duckworth, 2017). (Fig 1)
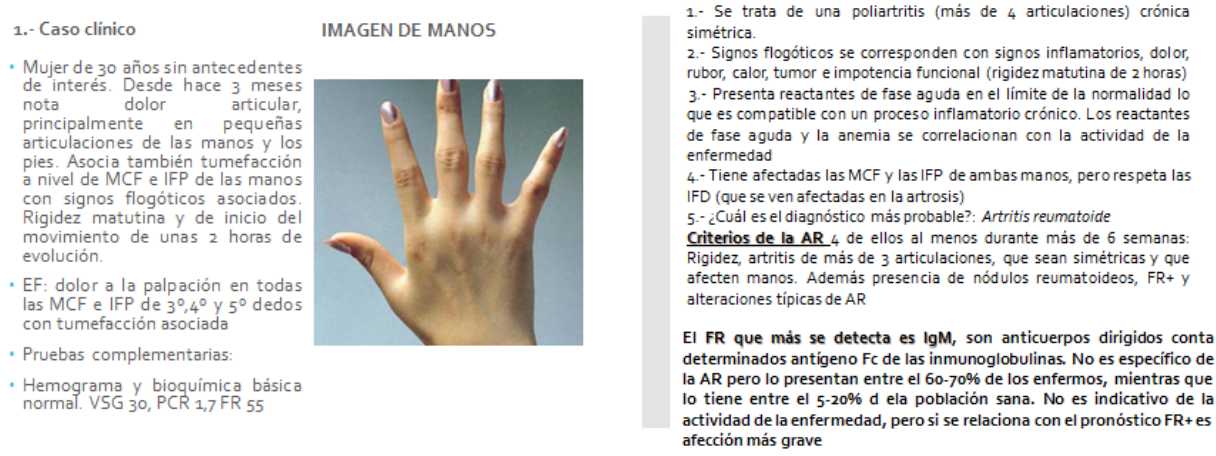

El FR que más se detecta es lgM, son anticuerpos dirigidos conta determinados antígeno $\mathrm{Fc}$ de las inmunoglobulinas. No es específico de la AR pero lo presentan entre el $60-70 \%$ de los enfermos, mientras que lo tiene entre el 5-20\% d ela población sana. No es indicativo de la actividad de la enfermedad, pero si se relaciona con el pronóstico FR+es afección más grave

Fig 1: Ejemplo de resolución de caso clínico. A) Caso clínico. B) Discusión

Los alumnos debían elaborar diagnósticos diferenciales, debían diferenciar tipos de dolor según descripción del paciente y comparar con criterios diagnósticos ya establecidos para cada entidad patológica (Manual SER, 2015). Se analizaba además la terminología científica empleada con el fin de familiarizar al alumnado con los términos científicos biosanitarios. Nuestro objetivo en cada sesión fue razonar el diagnóstico (Guías Fisterra, 2017). Terminado el caso clínico respondían a 4 o 5 preguntas tipo test relativas al mismo (Fig. 2).

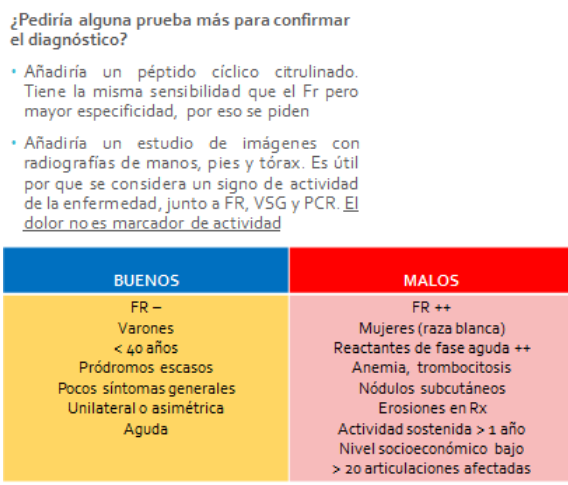

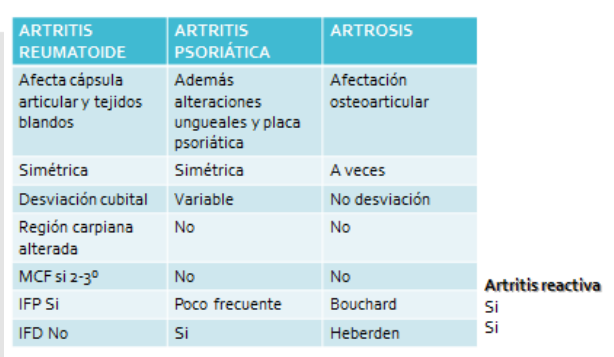

Fig 2: Ejemplo de resolución de caso clínico 
Transversalidad educativa: casos clínicos para el estudio de Geriatría y Patologías Osteoarticulares y su intervención en Autonomía e Independencia Funcional en el Adulto

\subsubsection{Descripción de la Metodología en Autonomía e Independencia Funcional en el Adulto}

AIF del adulto eligió dos casos a desarrollar uno de reumatología, concretamente mano reumática y otro de traumatología, una amputación. El caso de mano reumática, artritis reumatoide, se ha planteado en talleres:

1) Evaluación desde Terapia Ocupacional: perfil ocupacional, entrevista, herramientas de evaluación estandarizadas con la implementación de protocolos (DASH, MHOQ, HAQ, ARAT-KIT, etc.)

2) Técnicas de intervención: ejercicios de fortalecimiento, destreza y coordinación, economía articular, ergonomía, productos y tecnologías de apoyo, férulas, vendajes, terapia en espejo, entre otros. (Fig. 3)

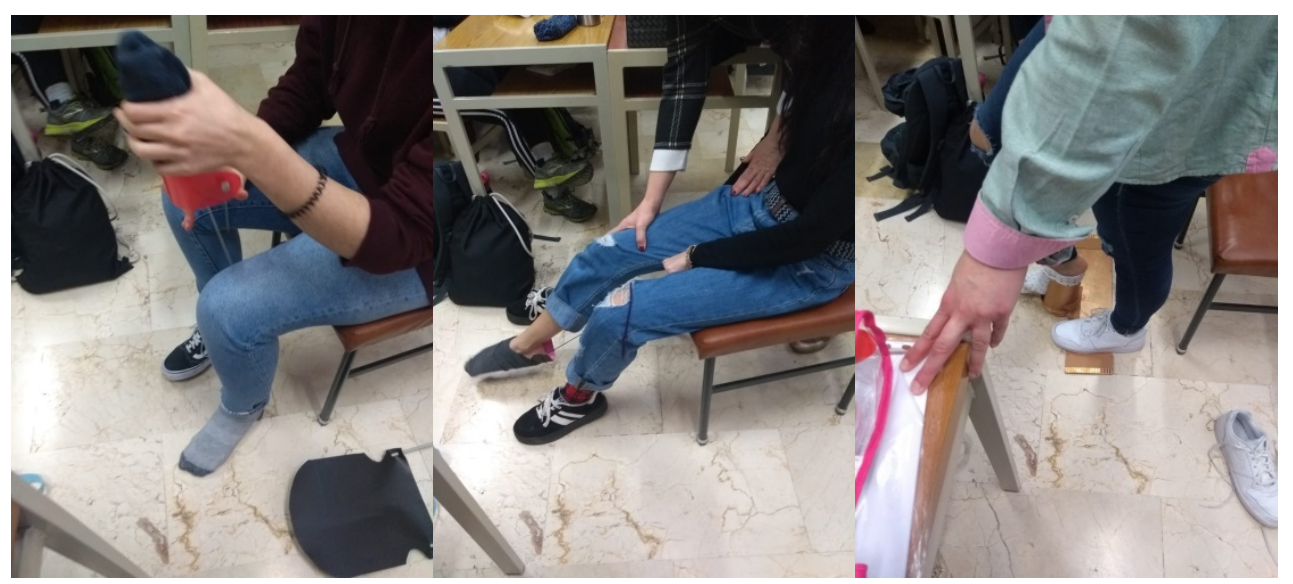

Fig. 3: Alumnos utilizando productos de apoyo de bajo coste de elaboración propia

3) Se realizaron dos talleres diseñados como simulación de discapacidad mediante vendajes limitadores de la capacidad funcional y posterior aplicación de intervenciones terapéuticas. Durante estos talleres una parte del alumnado simulaba la discapacidad esperable, por ejemplo en una paciente con mano afecta de artritis reumatoide y la otra parte simulaba la acción terapéutica, enseñándoles el uso de diferentes productos de apoyo. En otro taller, también relativo a técnicas de intervención se enseñó a los alumnos a confeccionar productos de apoyo de bajo coste (Fig. 4)

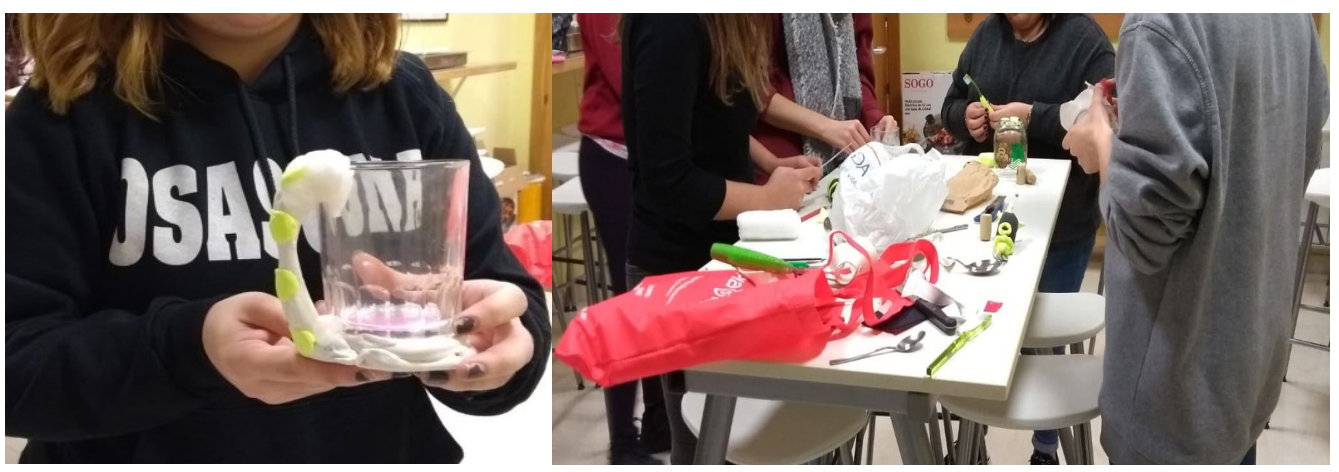

Fig 4: Alumnos diseñando productos de apoyo 
Por otro lado, se realizó un taller de los materiales de uso más común en ambas patologías y Terapia Ocupacional. Se utilizó la realización de una gamificación tipo scape room empleando diferentes casos y los materiales de uso más frecuente: digyflex, candos, therabands, masillas, y productos de apoyo para conservación de energía, entre otros (Fig. 5).
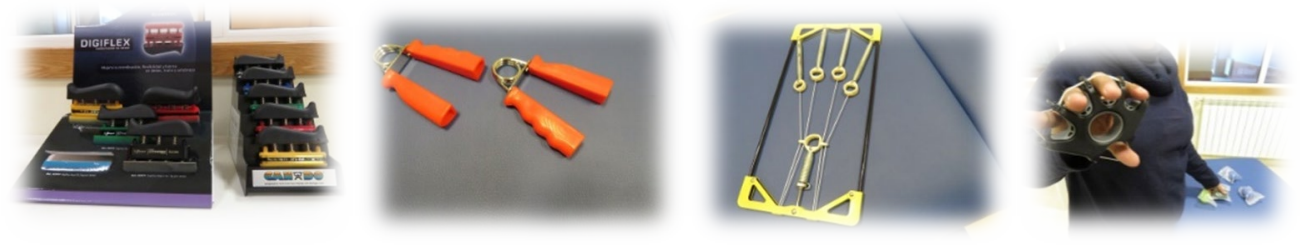

Fig 5.- Algunos materiales vistos durante las practicas de aula.

Además, en este taller se explicó y mostró la potencialidad de nuevas tecnologías tipo la impresora 3d a la hora de la creación de productos de apoyo de bajo coste (Fig. 6)

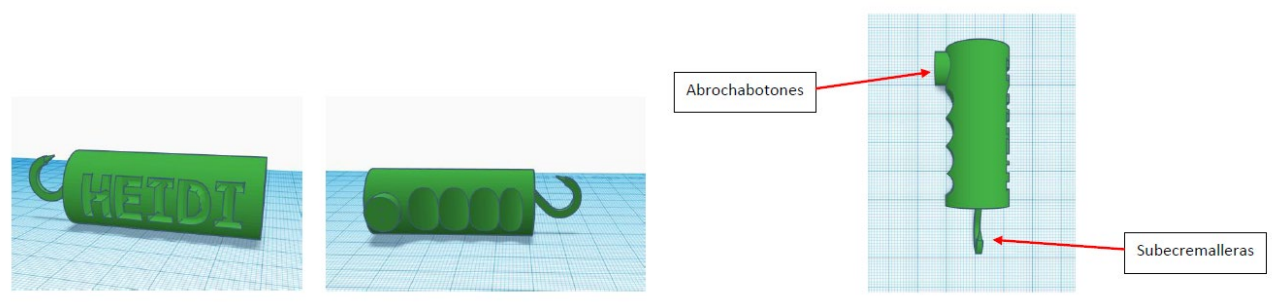

Fig 6.- Ejemplo de productos de apoyo para conservación de energía y patología osteoarticular realizados con la impresora $3 D$.

\section{Resultados}

\subsection{Valoración de indicadores}

En primer lugar comentar que la participación de los alumnos fue masiva en la resolución de casos clínicos, un $85 \%$ de participación. Es de reseñar que la actividad pertenecía la parte práctica de ambas asignaturas y de carácter obligatorio, si bien un pequeño porcentaje o bien no acudió esos días o bien su participación en la resolución de los casos clínicos fue muy escasa. La participación en AIF en el Adulto fue del 100\% del alumnado

En la asignatura de Geriatría y Patologías Osteoarticulares, los cuestionarios finales (preguntas de test) realizados en cada caso clínico obtuvieron un acierto global del $84 \%$ siendo en varios de ellos del $100 \%$. Todos se realizaban en la misma sesión e inmediatamente a la resolución del caso clínico.

En la convocatoria ordinaria de Geriatría y Patologías Osteoarticulares hemos detectado mayor número de alumnos no presentados con relación a años anteriores (41\%), creemos debido al calendario de exámenes y no a la dificultad añadida por los casos clínicos de la asignatura. En la convocatoria extraordinaria de mayo se presentaron el $93 \%$ de los convocados. En cuanto a las calificaciones obtenidas en convocatoria ordinaria no se aprecian diferencias significativas con relación a convocatorias anteriores, $71 \%$ aprobados. Se apreció un aumento de alumnos que superaron la materia con una calificación de notable sumando ambas convocatorias (Gráfico 1).

En lo referente a la asignatura AIF en el adulto, se concluye que en la convocatoria de mayo no sólo hay una mejora en la calificación media, puesto que en el curso 2017-2018 (aprobados=15; suspensos=19) la media de la asignatura fue de 5,94 $(0,73)$ mientras que en el 
Transversalidad educativa: casos clínicos para el estudio de Geriatría y Patologías Osteoarticulares y su intervención en Autonomía e Independencia Funcional en el Adulto

curso 2018-2019 (aprobados=13 y suspensos=12) la media de la asignatura fue de 6,3(1). Cabe señalar que este curso se obtuvieron 4 notables altos respecto al curso anterior que hubo dos notables bajos.

El grado de satisfacción fue medido con un cuestionario que los alumnos completaron en el caso de Geriatría y Patologías Osteoarticulares antes del examen en convocatoria ordinaria y en el caso de AIF en el Adulto al finalizar la resolución del primer caso clínico. El cuestionario se dividió en tres partes: evaluación de los casos clínicos, grado de dificultad y el grado de satisfacción general (Tabla 1 y 2).

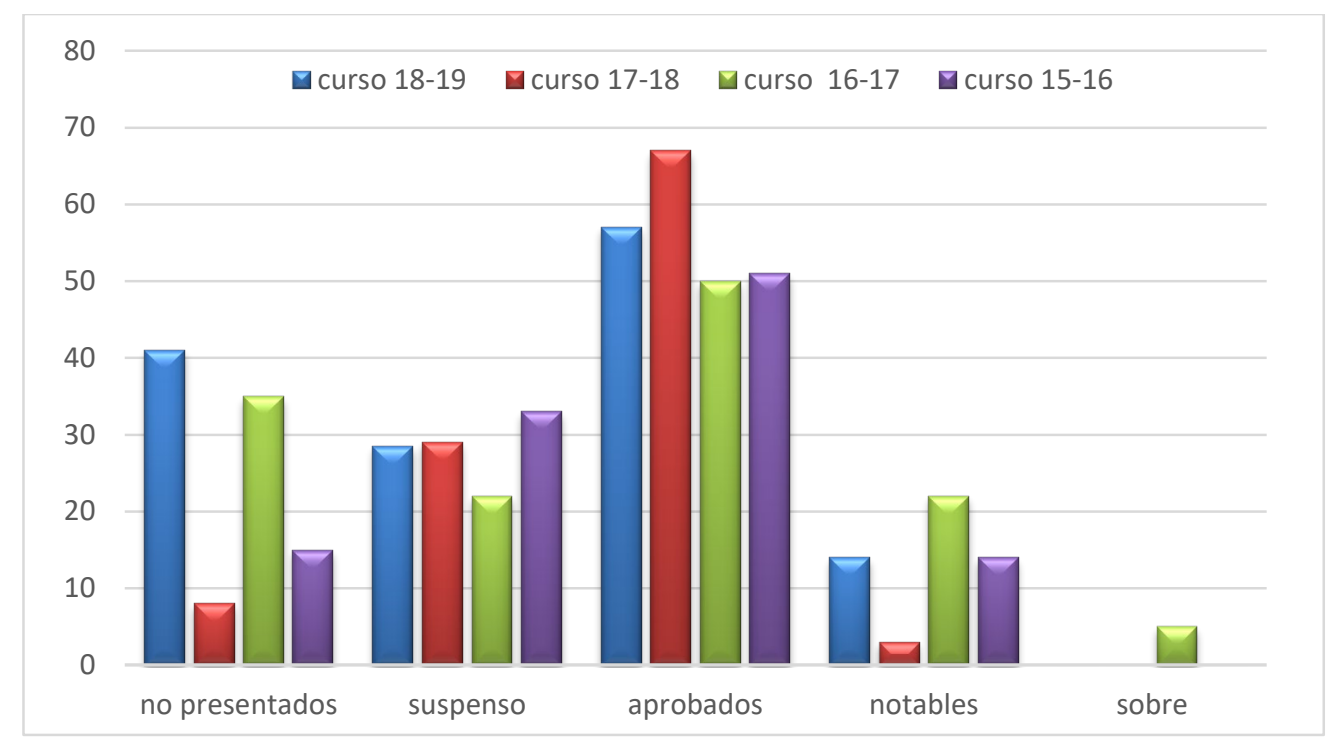

Gráfica 1: Representación de las calificaciones en convocatoria ordinaria de los últimos años, en porcentaje

En el apartado de la evaluación de los casos la media de valoración de los alumnos fue superior a 4 en ambas asignaturas. En el grado de dificultad la media obtenida fue de 3,5, también en ambas asignaturas. En el grado de satisfacción general se obtuvo un 4 en el interés de la materia y aumentar el conocimiento de la misma y 4,5 en la recomendación de seguir usándolo. Reconocen el 64\% de los alumnos que deberían haberlos trabajado más en Geriatría y Patologías Osteoarticulares y un $41 \%$ en AIF del Adulto.

Tabla 1: Encuesta de satisfacción del alumnado en Geriatría y Patologías Osteoarticulares

\begin{tabular}{|c|c|c|c|c|c|}
\hline \multicolumn{6}{|c|}{$\begin{array}{c}\text { ENCUESTA DE SATISFACCIÓN "siendo } 1 \text { totalmente en desacuerdo y } 5 \text { totalmente de } \\
\text { acuerdo" }\end{array}$} \\
\hline EVALUACIÓN CASOS CLÍNICOS & 1 & 2 & 3 & 4 & 5 \\
\hline 1 Considero que se ajusta a los contenidos teórico & & & & 4,3 & \\
\hline 2 El contenido de los mismo es claro y fácilmente entendible & & & 3,5 & & \\
\hline 3 Facilita el aprendizaje & & & & 4,07 & \\
\hline \multirow{2}{*}{$\begin{array}{l}4 \text { Es de utilidad en mi desempeño profesional } \\
\text { GRADO DE DIFICULTAD DE LOS CASOS CLÍNICOS }\end{array}$} & & & & 4,14 & \\
\hline & & & & & \\
\hline \multirow{2}{*}{$\begin{array}{ll}1 & \text { Me resultó difícil } \\
2 & \text { Me resultó ameno }\end{array}$} & & & 3,5 & & \\
\hline & & & & 4 & \\
\hline
\end{tabular}




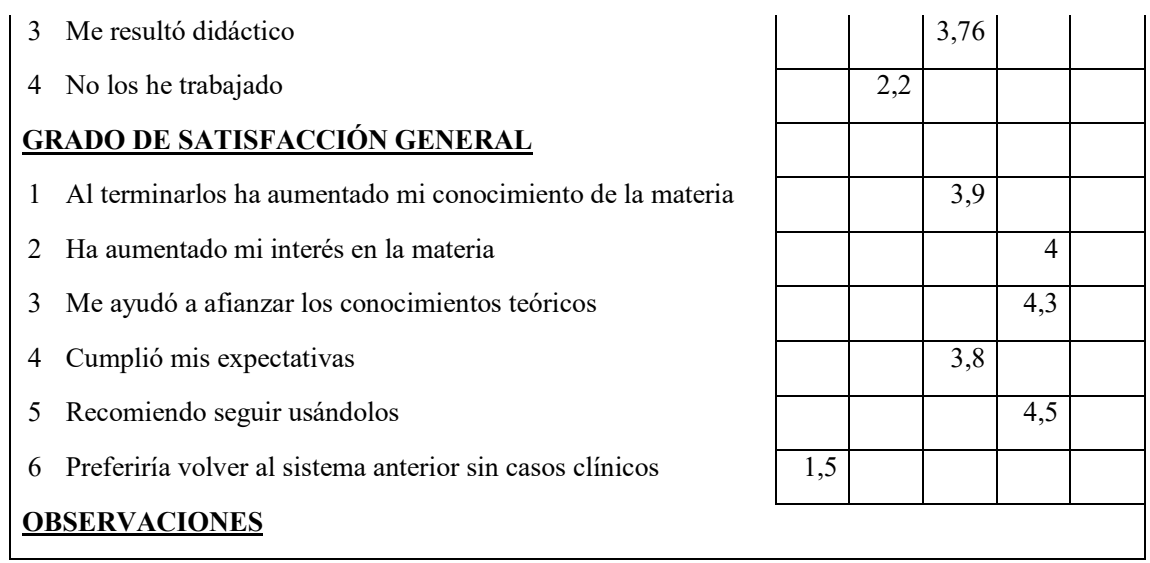

Tabla 2: Encuesta de satisfacción del alumnado en AIF en el Adulto

\begin{tabular}{|c|c|c|c|c|c|}
\hline \multicolumn{6}{|c|}{$\begin{array}{c}\text { ENCUESTA DE SATISFACCIÓN "siendo } 1 \text { totalmente en desacuerdo y } 5 \text { totalmente de } \\
\text { acuerdo" }\end{array}$} \\
\hline EVALUACIÓN CASOS CLÍNICOS & 1 & 2 & 3 & 4 & 5 \\
\hline \multirow{5}{*}{$\begin{array}{ll}1 & \text { Considero que se ajusta a los contenidos teórico } \\
2 & \text { El contenido de los mismo es claro y fácilmente entendible } \\
3 & \text { Facilita el aprendizaje } \\
4 & \text { Es de utilidad en mi desempeño profesional } \\
\text { GRADO DE DIFICULTAD DE LOS CASOS CLÍNICOS }\end{array}$} & & & & 4,4 & \\
\hline & & & 3,9 & & \\
\hline & & & & 4,2 & \\
\hline & & & & 4,4 & \\
\hline & & & & & \\
\hline 1 Me resultó difícil & & & 3,9 & & \\
\hline 2 Me resultó ameno & & & 3,1 & & \\
\hline 3 Me resulto didáctico & & & 3,6 & & \\
\hline 4 No los he trabajado & & 2,4 & & & \\
\hline \multicolumn{6}{|l|}{ GRADO DE SATISFACCIÓN GENERAL } \\
\hline 1 Al terminarlos ha aumentado mi conocimiento de la materia & & & 3,8 & & \\
\hline 2 Ha aumentado mi interés en la materia & & & 3,6 & & \\
\hline 3 Me ayudó a afianzar los conocimientos teóricos & & & 3,8 & & \\
\hline 4 Cumplió mis espectativas & & & 3,1 & & \\
\hline 5 Recomiendo seguir usándolos & & & & 4 & \\
\hline 6 Preferiría volver al sistema anterior sin casos clínicos & 1,75 & & & & \\
\hline OBSERVACIONES & & & & & \\
\hline
\end{tabular}

\subsection{Resumen de resultados}

Este proyecto nos ha permitido mayor coordinación entre materias transversales dando enfoque eminentemente práctico al conocimiento evitando reiteraciones de materia y acercando al alumno a la realidad de su profesión.

Hemos mantenido los resultados académicos globales con relación a cursos anteriores y mejorado las calificaciones, número de notables (Tabla 3). La participación del alumnado 
cumplió las expectativas y el grado de satisfacción fue elevado tanto por parte del alumnado como por el equipo docente.

Todo el material elaborado está en la plataforma on line con el consiguiente uso de medios

Tabla 3: Resumen de resultados de indicadores propuestos

\begin{tabular}{|c|c|c|c|}
\hline $\mathbf{N}^{\mathbf{0}}$ & Indicador & Modo de evaluación & Rangos fijados y obtenidos \\
\hline 1 & $\begin{array}{l}\text { Número de alumnos que } \\
\text { solucionen correctamente } \\
\text { los casos clínicos en el } \\
\text { tiempo establecido }\end{array}$ & $\begin{array}{l}\text { Respuestas correctas a los cuestionarios de cada } \\
\text { caso }\end{array}$ & $\begin{array}{l}\text { 0\%-40\% Bajo } \\
41 \%-80 \% \text { aceptable } \\
>80 \% \text { bueno } \\
\text { Obtuvo } 84 \% \text { BUENO }\end{array}$ \\
\hline 2 & $\begin{array}{l}\text { Número de aprobados } 2018 \text { - } \\
2019 \text { en Geriatría y } \\
\text { Patología Osteoarticular }\end{array}$ & $\begin{array}{l}\text { Se tomarán como indicadores las notas de } \\
\text { convocatoria ordinaria }\end{array}$ & $\begin{array}{l}0-50 \% \text { bajo } \\
51-70 \% \text { aceptable } \\
\geq 71 \% \text { bueno } \\
\text { Obtuvo } 71 \% \text { BUENO }\end{array}$ \\
\hline 3 & $\begin{array}{l}\text { Número de aprobados 2018- } \\
2019 \text { en AIF del adulto }\end{array}$ & $\begin{array}{l}\text { Se tomarán como indicadores las notas de } \\
\text { convocatoria ordinaria de mayo }\end{array}$ & $\begin{array}{l}\text { 0-50\% bajo } \\
51-70 \% \text { acceptable } \\
\geq 71 \% \text { bueno } \\
\text { Obtuvo }>\mathbf{5 1 \%} \text { ACEPTABLE }\end{array}$ \\
\hline 4 & $\begin{array}{l}\text { Grado de satisfacción del } \\
\text { alumno }\end{array}$ & Encuesta realizada antes de la evaluación & $\begin{array}{l}3,8 \text { sobre } 5 \text { POA } \\
3,7 \text { sobre } 5 \text { AIF }\end{array}$ \\
\hline
\end{tabular}

\section{Conclusiones}

Las referencias bibliográficas sobre el uso de casos clínicos en docencia que hemos localizado y utilizadas como justificación para nuestro proyecto no contemplan la Terapia Ocupacional, sin embargo la petición mayoritaria de nuestros egresados (Informe de Encuesta de Egresados curso 2016-2017) es dar valor a las prácticas desde el primer momento de ingreso en el Grado.

Los casos clínicos son utilizados de manera habitual en otros grados sanitarios, por ejemplo en el Grado de Medicina, siendo inusual en el nuestro. Creemos que es necesario acercar al alumnado a la realidad profesional del mundo sanitario y al contexto que supone trabajar con personas con discapacidad. La gamificación de las aulas está siendo utilizada para facilitar la implicación del alumnado con el uso de nuevas tecnologías (XI JiD, 2019)

Hemos realizado una labor de coordinación transversal entre materias del mismo curso lo cual facilita y refuerza el aprendizaje y esa labor se ha realizado de forma práctica, recortándose el tiempo dedicado a clase expositiva (Norniella, 2019). Destacamos la implicación del alumnado y el grado de satisfacción del mismo, así como la valoración cualitativa de estos.

Hemos encontrado mejoras en las calificaciones finales tomando como referencia convocatoria ordinaria y extraordinaria en Geriatría y Patologías Osteoarticulares. En convocatoria ordinaria no se observó mejoría alguna, aunque sí en AIF Adulto, por lo que tendremos que replantear contenidos y prácticas de aula y laboratorio para mejorar ese parámetro. Debemos mejorar, así mismo, la parte técnica con incorporación de nuevas tecnologías, por ejemplo, talleres de 
estrategias de intervención incluyendo tecnologías de apoyo y utilizar códigos QR en la solución de los casos clínicos de Geriatría y Patologías Osteoarticulares.

Pensamos seguir con aplicando esta metodología alojando nuevos recursos generados durante las clases y talleres en la plataforma online para disposición de todos los alumnos del Grado en Terapia Ocupacional

\section{Bibliografía}

ARthanAt, S., \& LenKER, J. (2004). Evaluating the ICF as a Framework for Clinical Assesment of Persons for Assistive Technology Devise Recommendaion, 10 North American Collaborating Centre (NACC). Conference on ICF, Halifax.ASEM.

Buckup, K y Buckup, J. (2013). Pruebas clínicas para patología ósea, articular y muscular. Elsevier $5^{\mathrm{a}} \mathrm{Ed}$.

College of Occupational Therapist (2004). Guidance for the Use of the International Classification of Functioning, Disability and Health (ICF) and the Ottawa Charter for Health Promotion in Occupational Therapy Services. London: College of Occupational Therapist.

Cruz, J., SAStres, I., \& Romero, S. (2018). Hoja de información al paciente y consentimiento informado de casos clínicos y series de casos: propuesta de un modelo estandarizado para comunicaciones en congresos y otras publicaciones científicas. Reumatologia Clinica 14 (4) 181-250.

Duckworth, A., Ponter, D., \& Rainston, S. (2017). Ortopedia, traumatología y reumatología. Elsevier

Firestein, G., Budd, G., Grabriel, S., Mcinnes, I. \& O’Dell, J. (2018). Kelley y Firestein. Tratado de reumatología, Elservier $10^{\mathrm{a}}$ ed.

GonZÁLEZ, J. (2018). Ludificación en el aula: experiencia basada en actividad “escape room”. En : XI Jornadas de Innovación Docente 2018. Universidad de Oviedo (2019, Mieres. Asturias)

GUÍAS FISTERRA. www.fisterra.com/guias-clinicas/

LENKER, J.A., \& JutAI, J.W. (2002). Assistive technology research and practice: What role for ICF? Paper presented at the 8 th North American Collaboratin Center (NACC) Conference on ICF, Toronto, ON.

XI JoRnADAS DE INNOVACión Docente 2018. Mesa redonda "Experiencias que promueven la coordinación entre profesorado, la experiencia en tutorización y estragias de evaluación. 2019, Mieres. Asturias

OMS (2001). Clasificación Internacional del Funcionamiento de la Discapacidad y de la Salud.

Pineda, C., \& Sandoval, H. (2018). Protocolos para publicación de casos clínicos. Reumatología Clinica, 14.

SOCIEDAD ESPAÑOLA DE REUMATOLOGÍA (2015). Manual Ser de enfermedades reumáticas. Elsevier. 